\title{
Anterior Cruciate Ligament Reconstruction with LARS Artificial Ligament-Clinical Results after a Long-Term Follow-Up
}

\author{
Paolo Domenico Parchi ${ }^{1}$ Gianluca Ciapini ${ }^{1} \quad$ Carlo Paglialunga $^{1} \quad$ Michele Giuntoli $^{1}$ Carmine Picece ${ }^{1}$ \\ Fabio Chiellini ${ }^{1}$ Michele Lisanti ${ }^{1}$ Michelangelo Scaglione ${ }^{1}$
}

1 1st Orthopedic Division of Pisa University, Department of
Translational Research and New Technologies in Medicine and
Surgery, University of Pisa, Pisa, Italy

Joints 2018;6:75-79.

\author{
Address for correspondence Paolo Domenico Parchi, MD, PhD, 1st \\ Orthopedic Division of Pisa University, Department of Translational \\ Research and New Technologies in Medicine and Surgery, University \\ of Pisa, via Paradisa 2, Pisa 56100, Italy \\ (e-mail: paolo.parchi@unipi.it).
}

\begin{abstract}
Keywords

- anterior cruciate ligament

- reconstruction

- ligament reinforcement

- artificial ligament

- LARS ligament

Purpose The aim of this retrospective study was to evaluate the subjective and functional outcome of anterior cruciate ligament (ACL) reconstruction with the synthetic Ligament Advanced Reinforcement System (LARS) ligament.

Methods Twenty-six patients were reviewed at an average follow-up of 11.6 years. Objective clinical evaluation was performed with stability tests. Patient-reported outcomes (Visual Analogue Scale, Knee Injury and Osteoarthritis Outcome Score, and Cincinnati Knee Rating Scale) were used to assess subjective and functional outcomes.

Results Overall satisfactory results were obtained in 22 cases (84.6\%). Four patients (15.4\%) showed mechanical failure of the graft. No cases of synovitis or infection were reported.

Conclusion LARS ligament can be considered a safe and suitable option for $\mathrm{ACL}$ reconstruction in carefully selected cases, especially elderly patients needing a rapid postoperative recovery.

Level of Evidence Level IV, retrospective case series.
\end{abstract}

\section{Introduction}

Four-strand hamstring tendons or bone-patellar tendonbone are the most commonly used autograft and actually represent the gold standard for the anterior cruciate ligament (ACL) reconstruction. ${ }^{1,2}$ The main complications reported with the use of tendon autografts are related to graft harvesting and donor site morbidity. ${ }^{3}$

Synthetic grafts have been proposed to overcome the problems related to the use of autografts. The first attempt to use an artificial ligament for ACL reconstruction was made in 1918 by Smith, who proposed a graft made up of multiple silk sutures fastened with staples. ${ }^{4}$ Unfortunately, the procedure was unsuccessful with a complete graft failure within the first 3 months from the implantation.

received

August 21, 2017

accepted after revision

April 8, 2018

published online

May 23, 2018
DOI https://doi.org/

10.1055/s-0038-1653950. ISSN 2282-4324.
Between 1970s and 1980s, there was a progressive new interest in the use of artificial ligaments, supported by the development of new materials and new devices such as Teflon, carbon fibers, Gore-Tex, Dacron, and subsequently Leeds-Keio and Kennedy-ligament augmentation device (LAD). During the years, these materials have all been abandoned due to the high rate of complications related with their use, like reactive synovitis and graft breakage in short or medium term, and in the 1990s the use of synthetic grafts was completely abandoned..$^{5-8}$

In the last years of 1990s, a new generation of synthetic graft for ligament reconstruction has been proposed, with the introduction of the Ligament Advanced Reinforcement System (LARS; LARS Company, Arc sur Tille, France). Despite being introduced in the market since several years ago, it is
Copyright (c) 2018 Georg Thieme Verlag License terms

KG Stuttgart · New York 
not widely used yet. ${ }^{9}$ This is probably due to the small number of long-term studies and the negative previous experiences with the last generation of synthetic ligaments.

The aim of this study was to evaluate the safety and the efficacy of ACL reconstruction with the LARS ligament at long-term follow-up. The hypothesis of the study was that ACL reconstruction with the LARS artificial ligament is a safe and effective procedure.

\section{Methods}

\section{Participants}

This study was designed as a retrospective analysis of patients who have undergone primary, isolated ACL reconstruction using the LARS ligament in a single institution between 2003 and 2005. The study was authorized by the internal revision board and was performed in accordance with the ethical standards of the 1964 Declaration of Helsinki as revised in 2000 .

The use of a synthetic graft for the ACL reconstruction was proposed to all patients older than 30 years with a symptomatic isolated ACL injury that required a quick return to their previous sport activity level as a possible alternative to the autograft. All patients were clearly informed about the advantages and the possible complications related to the use of a synthetic graft.

All patients gave informed consent before being included into the study.

\section{Interventions}

All the procedures were performed under regional anesthesia by the same surgeon. In all cases, the ACL reconstruction was performed with an arthroscopically assisted technique. Two types of LARS ligament were used: the AC50DB with a strength of 2,300 Newton and a diameter of $5 \mathrm{~mm}$ and the AC40DB with a strength of 1,700 Newton and a diameter of $4 \mathrm{~mm}$. The choice between these two types of LARS was related mainly to the patients' weight (we used the AC50DB for patients weighing more than $80 \mathrm{~kg}$ ).

The LARS ligament was placed into the native femoral and tibial ACL footprint. The tibial tunnel was created using a cannulated reamer matching the diameter of the graft ( $7.5 \mathrm{~mm}$ for the AC40DB and $8 \mathrm{~mm}$ for the AC50DB). The tibial guide was angled at $55^{\circ}$ to the tibial plateau. The femoral reamer was, then, introduced through the tibial tunnel at $90^{\circ}$ of knee flexion, and the half femoral tunnel was reamed under arthroscopic control reaching a depth of 35 to $40 \mathrm{~mm}$. The doubled LARS ligament was then introduced and fixed to the femur using a suspensory crosspin fixation device (Transfix; Arthrex, Naples, Florida, United States) and to the tibia using a metal interference screw.

Postoperative rehabilitation started from the first postoperative day by performing active and passive knee range of motion (ROM) exercises. Patients were discharged on the second postoperative day and partial weight-bearing on the operated limb was allowed for 2 weeks. After that period, patients were progressively allowed to increase the weightbearing reaching the full weight-bearing 4 weeks after surgery.

\section{Outcome Measures}

All the patients were clinically evaluated for pain, swelling, stability, and ROM. Knee stability was tested using the Rolimeter knee tester (Aircast; DJO Global, Vista, California, United States $)^{10}$ for the anterior drawer test and the Lachman test. Both tests were performed three times on both knees and the average side-to-side (SSD) difference at maximal manual traction was recorded. Instrumental evaluation of anterior knee stability was scored as excellent (SSD $<2 \mathrm{~mm}$ ), good $(\mathrm{SSD}=2-4 \mathrm{~mm})$, or poor $(\mathrm{SSD}>4 \mathrm{~mm})$.

Subjective and functional evaluation was based on three patient-reported outcomes (PROs): the Visual Analogue Scale (VAS), the Knee Injury and Osteoarthritis Outcome Score (KOOS), and the Cincinnati knee rating scales.

The VAS is used to quantify the pain perceived by the patient on the affected limb.

The KOOS is a PRO measurement instrument, developed to assess the patients' opinion about their knee and associated problems. The KOOS evaluates both short-term and long-term consequences of knee injury including ACL ligament injuries. It holds 42 items in 5 separately scored subscales: pain, symptoms, function in daily living (KOOS-ADL), function in sport and recreation (KOOS-Sport/Rec), and knee-related quality of life (KOOS-QOL). ${ }^{11}$

The Cincinnati knee rating scales evaluates eight subjective parameters (pain, swelling, giving way, overall activity level, walking, stairs, running, jumping, or twisting) to assess the patient's functional recovery. ${ }^{12}$

\section{Results}

From January 2003 to December 2005, we performed 29 ACL reconstructions using the LARS ligament. The mean age of patients was 38.5 years (range, 32-52 years). Gender distribution was 20 males and 6 females. Average time between injury and surgery was 2 months (range, 1-5 months). All patients returned to sports activities after an average of 4 months (range, 1.5-7 months).

Three patients were lost at the follow-up, but we were unable to establish the reasons for their loss, because we could not contact them. The remaining 26 patients were examined at an average follow-up of 139.3 months (range, 129-154 months).

We recorded four cases of mechanical graft failure that occurred after a new sports injury. In one case, it occurred after 4 years from the implantation and in three cases over 10 years after surgery ( - Fig. $\mathbf{1}$ ). All the cases of LARS failure were documented by a magnetic resonance imaging (MRI) evaluation. Only two cases required a surgical revision for a persistent knee instability. The torn graft was removed arthroscopically and replaced with a new LARS ligament using the same surgical technique. In the other two cases, the MRI showed graft breakage, but the patients did not suffer from knee instability.

Clinical assessment did not reveal signs of graft-related problems like knee reactive synovitis. Absence of reactive knee synovitis was also confirmed by MRI evaluations that were available at follow-up in 15 patients. 

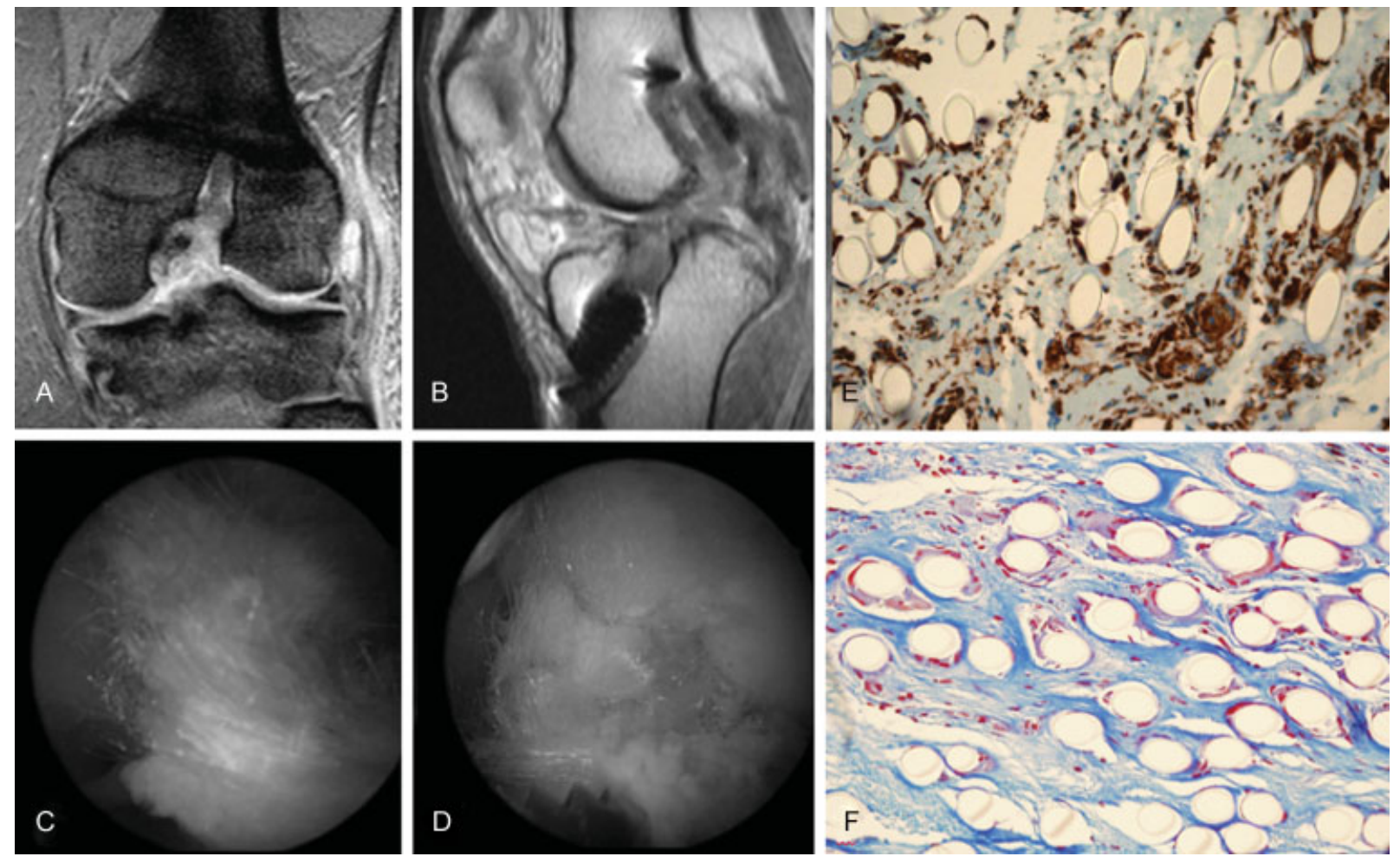

Fig. 1 Mechanical graft failure occurred at 4 years in a 38-year-old man during sport activity (soccer). (A, B) Magnetic resonance imaging evaluation showed Ligament Advanced Reinforcement System rupture without signs of knee synovitis. (C, D) Intraoperative findings. (E) Immunostaining with anti-vimentin antibody that shows connective tissue, fibroblastic cells, and multinucleated giant cells around the polyethylene terephthalate (PET) fibers. (F) Masson's trichrome stain that shows connective tissue, fibroblastic cells, and multinucleated giant cells around the PET fibers.

ROM of the treated knee did not differ from the contralateral one in 15 patients (57.7\%). Loss of knee flexion of less than $10^{\circ}$ was observed in 7 patients $(26.9 \%)$ and between $10^{\circ}$ and $15^{\circ}$ in 4 patients $(15.4 \%)$.

Instrumented analysis of anterior knee laxity with the Rolimeter knee tester showed an excellent result (SSD $<2 \mathrm{~mm}$ ) in 15 patients (57.7\%), good (SSD $=2-4 \mathrm{~mm}$ ) in 7 cases $(26.9 \%)$, and poor (SSD $>4 \mathrm{~mm}$ ) in 4 cases (15.4\%).

The mean VAS was 1.69 . Score distribution was 0 to 2 points in 17 patients (65.4\%), 3 to 5 points in 6 patients (23.1\%), and 6 to 8 points in 3 patients (11.5\%).

Global mean KOOS was 80 with 13 (50\%) excellent results, 9 good results (34.6\%), and 4 poor result (15.4\%) (-Table 1 ).

Functional evaluation with the Cincinnati knee rating scale showed a mean score of 84.5 points (range, 53-100 points) with 15 excellent results (57.7\%), 7 good results (26.9\%), and 4 poor results (15.4\%).

\section{Discussion}

Artificial substitutes of ACL gained popularity in the 1970s and 1980 s, but the high failure rate (reactive synovitis and mechanical breakdown) led to a complete stop in their use in the 1990 s. ${ }^{13}$ During the past 15 years, the development of biocompatible materials and a better understanding of the knee kinematics led to the development of a new generation of synthetic grafts. ${ }^{9}$
The LARS ligament consists of fibers made of polyethylene terephthalate (PET) composed of two parts: an intraosseous segment composed of longitudinal fibers bound together by a transverse knitted structure and an intra-articular segment composed of parallel longitudinal free fibers twisted at $90^{\circ}$. The PET fibers of the intra-articular segment are designed to encourage tissue ingrowth due to the porosity of the material, allowing ingrowth from the surrounding bony tunnels as shown from in vitro studies. ${ }^{14}$

Table 1 KOOS results

\begin{tabular}{|l|l|l|l|l|}
\hline & $\begin{array}{l}\text { Mean value } \\
\text { (range) }\end{array}$ & $\begin{array}{l}\text { Excellent } \\
>90 \\
N(\%)\end{array}$ & $\begin{array}{l}\text { Good } \\
70-89 \\
N(\%)\end{array}$ & $\begin{array}{l}\text { Poor } \\
<69 \\
N(\%)\end{array}$ \\
\hline Symptoms & $83(35-100)$ & $14(53.8)$ & $8(30.8)$ & $4(15.4)$ \\
\hline Pain & $89(66-100)$ & $16(61.5)$ & $8(30.8)$ & $2(7.7)$ \\
\hline $\begin{array}{l}\text { Function } \\
\text { daily living }\end{array}$ & $83(59-100)$ & $16(61.5)$ & $6(23.1)$ & $4(15.4)$ \\
\hline $\begin{array}{l}\text { Sport/ } \\
\text { recreation } \\
\text { function }\end{array}$ & $82(45-100)$ & $13(50)$ & $7(26.9)$ & $6(23.1)$ \\
\hline $\begin{array}{l}\text { Quality } \\
\text { of life }\end{array}$ & $77(18-100)$ & $10(38.4)$ & $8(30.8)$ & $8(30.8)$ \\
\hline Global & $80(53-100)$ & $13(50)$ & $9(34.6)$ & $4(15.4)$ \\
\hline
\end{tabular}

Abbreviation: KOOS, Knee Injury and Osteoarthritis Outcome Score. 
The first clinical report on the use of LARS in ACL reconstruction was made by Dericks in $1995 .{ }^{15}$ He reported encouraging results in 220 cases with a mean follow-up of 2.5 years, with no cases of knee synovitis.

In a previous work published in 2013, we made a literature review of all the papers that reported clinical results of the LARS ligament for knee ligament reconstruction, searching in four different databases (Pubmed, Scopus, Embase, and Google Scholar). ${ }^{9}$ We found 35 papers for a total number of 1,245 cases with a follow-up ranging from 3 months to 9 years. We focused our attention on the number of graft ruptures and the number of cases of knee synovitis that were complications historically related to the use of artificial ligaments. We recorded 12 graft ruptures $(0.96 \%)$ and only 3 cases of knee synovitis $(0.24 \%){ }^{9}$

These data are confirmed by the results of this study and by two recent systematic reviews. ${ }^{16,17}$ Anderson et $\mathrm{al}^{16}$ reported comparable outcomes between the use of LARS ligament and the use of autografts after a medium-term follow-up. Batty et $\mathrm{a}^{17}$ compared the LARS ligament and others artificial ligaments (Leeds-Keio, Kennedy-LAD, Dacron, Gore-Tex, and Trevira) in terms of complications and clinical results. The authors reported the lowest cumulative rates of failure with the LARS device $(2.6 \%$ for ACL and $1 \%$ for posterior cruciate ligament surgery). Rates of noninfective synovitis and effusion ranged from $0.2 \%$ for LARS to $27.6 \%$ for Gore-Tex. Revision rates ranged from $2.6 \%$ for LARS to $11.8 \%$ for Trevira.

Although the LARS ligament has been used for ACL reconstruction since the late 1990s, there are very few studies that analyzed the long-term results and sometimes the reported data are discordant. ${ }^{18-21}$ Cerulli et $\mathrm{al}^{18}$ reported the results obtained with the use of LARS ligament in ACL reconstruction in a group of 25 patients after 9 years of follow-up. The authors reported satisfactory results in over $95 \%$ of the cases with no cases of knee synovitis. Jia et $\mathrm{al}^{19}$ reported the results of 91 patients at 7-year follow-up. They reported a failure rate of $4.4 \%$, and an overall complication rate of $2.2 \%$ with $86.6 \%$ of the patients returning to their sports activities. Wang et $\mathrm{al}^{20}$ reported on 26 cases of ACL reconstruction with LARS after a mean follow-up of 11.9 years. The authors reported a complication rate of $28.6 \%$ with two cases of knee synovitis. In this cases series, one of the highlighted causes of graft failure was the loosening of the fixation system. Tiefenboeck et $\mathrm{al}^{21}$ reported a low rate of patient satisfaction (55.6\%) and a high rate of complications (56\%) after a minimum follow-up of 10 years. Complications consisted of graft breakage during sport activity in 3 cases, surgical site infection in 3 cases, and residual knee instability in 4 cases. The authors did not report cases of knee reactive synovitis.

Differences between this study and that by Tiefenboeck et $\mathrm{al}^{21}$ might be related to the method of graft fixation method. As reported by Viateau et $\mathrm{al}^{22}{ }^{22}$ the LARS ligament has a very poor capacity of osteointegration in the bone tunnels. For this reason, fixation should not be achieved with absorbable devices.

To overcome the problem of the LARS ligament's osteointegration, several authors worked in the last years to enhance the biologic fixation of the graft using nanotechnologies. ${ }^{23-26}$
Another important way to reduce the risk of long-term failure and debris formation that can led to reactive knee synovitis is to preserve the ACL stumps, as suggested by Trieb et al. ${ }^{14}$ Preservation of the torn ligament reduces the friction stress on the LARS fibers at the level of the bone tunnels and between the fibers themselves and promotes fibroblast ingrowth.

This study has some limitations, mainly related to its retrospective design. Moreover, the sample size is small and all the patients were enrolled at the same hospital and operated by the same surgeon. Finally, no baseline outcome measures were available and therefore no data analysis on improvement of baseline conditions was possible.

In conclusion, even if the long-term results of the use of LARS are encouraging, the use of autologous grafts remain the gold standard in ACL reconstruction, especially in young people. LARS ligament can be considered a suitable option for ACL reconstruction in carefully selected cases, especially for older patients needing a fast functional recovery.

\section{Conflict of Interest}

None.

\section{References}

1 Budny J, Fox J, Rauh M, Fineberg M. Emerging trends in anterior cruciate ligament reconstruction. J Knee Surg 2017;30(01): 63-69

2 Kim HS, Seon JK, Jo AR. Current trends in anterior cruciate ligament reconstruction. Knee Surg Relat Res 2013;25(04):165-173

3 Samuelsson K, Andersson D, Karlsson J. Treatment of anterior cruciate ligament injuries with special reference to graft type and surgical technique: an assessment of randomized controlled trials. Arthroscopy 2009;25(10):1139-1174

4 Smith SA. The diagnosis and treatment of injuries to the crucial ligaments. Br J Surg 1918;6(22):176-189

5 Bolton CW, Bruchman WC. The GORE-TEX expanded polytetrafluoroethylene prosthetic ligament. An in vitro and in vivo evaluation. Clin Orthop Relat Res 1985;(196):202-213

6 Richmond JC, Manseau CJ, Patz R, McConville O. Anterior cruciate reconstruction using a Dacron ligament prosthesis. A long-term study. Am J Sports Med 1992;20(01):24-28

7 Rading J, Peterson L. Clinical experience with the Leeds-Keio artificial ligament in anterior cruciate ligament reconstruction. A prospective two-year follow-up study. Am J Sports Med 1995;23(03):316-319

8 Fujikawa K, Iseki F, Seedhom BB. Arthroscopy after anterior cruciate reconstruction with the Leeds-Keio ligament. J Bone Joint Surg Br 1989;71(04):566-570

9 Parchi PD, Gianluca C, Dolfi L, et al. Anterior cruciate ligament reconstruction with LARS ${ }^{\mathrm{TM}}$ artificial ligament results at a mean follow-up of eight years. Int Orthop 2013;37(08):1567-1574

10 Schuster AJ, McNicholas MJ, Wachtl SW, McGurty DW, Jakob RP. A new mechanical testing device for measuring anteroposterior knee laxity. Am J Sports Med 2004;32(07):1731-1735

11 Collins NJ, Misra D, Felson DT, Crossley KM, Roos EM. Measures of knee function: International Knee Documentation Committee (IKDC) Subjective Knee Evaluation Form, Knee Injury and Osteoarthritis Outcome Score (KOOS), Knee Injury and Osteoarthritis Outcome Score Physical Function Short Form (KOOS-PS), Knee Outcome Survey Activities of Daily Living Scale (KOS-ADL), Lysholm Knee Scoring Scale, Oxford Knee Score (OKS), Western Ontario and McMaster Universities Osteoarthritis Index (WOMAC), Activity Rating Scale (ARS), and Tegner Activity Score (TAS). Arthritis Care Res (Hoboken) 2011;63(Suppl 11):S208-S228 
12 Barber-Westin SD, Noyes FR, McCloskey JW. Rigorous statistical reliability, validity, and responsiveness testing of the Cincinnati knee rating system in 350 subjects with uninjured, injured, or anterior cruciate ligament-reconstructed knees. Am J Sports Med 1999;27(04):402-416

13 Savarese A, Lunghi E, Budassi P, Agosti A. Remarks on the complications following ACL reconstruction using synthetic ligaments. Ital J Orthop Traumatol 1993;19(01):79-86

14 Trieb K, Blahovec H, Brand G, Sabeti M, Dominkus M, Kotz R. In vivo and in vitro cellular ingrowth into a new generation of artificial ligaments. Eur Surg Res 2004;36(03):148-151

15 Dericks G Jr. Ligament advanced reinforcement system anterior cruciate ligament reconstruction. Oper Tech Sports Med 1995; 3:187-205

16 Anderson MJ, Browning WM III, Urband CE, Kluczynski MA, Bisson LJ. A systematic summary of systematic reviews on the topic of the anterior cruciate ligament. Orthop J Sports Med 2016;4(03): 2325967116634074

17 Batty LM, Norsworthy CJ, Lash NJ, Wasiak J, Richmond AK, Feller JA. Synthetic devices for reconstructive surgery of the cruciate ligaments: a systematic review. Arthroscopy 2015;31(05):957-968

18 Cerulli G, Antinolfi P, Bruè S, et al. Esperienza clinica nell'uti- lizzo di biomateriali nel ginocchio. GIOT 2011;37(Suppl 1):159-166

19 Jia Z, Xue C, Wang W, Liu T, Huang X, Xu W. Clinical outcomes of anterior cruciate ligament reconstruction using LARS artificial graft with an at least 7-year follow-up. Medicine (Baltimore) 2017;96(14):e6568
20 Wang C-L, Hsiao C-K, Ku M-C, Chang C-H. Arthroscopic anterior cruciate ligament reconstruction with LARS artificial ligament: an 8-15-year follow-up. J Mech Med Biol 2013;13(02): 1350046

21 Tiefenboeck TM, Thurmaier E, Tiefenboeck MM, et al. Clinical and functional outcome after anterior cruciate ligament reconstruction using the LARS ${ }^{\mathrm{TM}}$ system at a minimum follow-up of 10 years. Knee 2015;22(06):565-568

22 Viateau V, Manassero M, Anagnostou F, Guérard S, Mitton D, Migonney V. Biological and biomechanical evaluation of the ligament advanced reinforcement system (LARS AC) in a sheep model of anterior cruciate ligament replacement: a 3-month and 12-month study. Arthroscopy 2013;29(06):1079-1088

$23 \mathrm{Li} \mathrm{H}$, Chen S, Wu Y, et al. Enhancement of the osseointegration of a polyethylene terephthalate artificial ligament graft in a bone tunnel using 58S bioglass. Int Orthop 2012;36(01):191-197

$24 \mathrm{Li} \mathrm{H}, \mathrm{Ge}$ Y, Wu Y, et al. Hydroxyapatite coating enhances polyethylene terephthalate artificial ligament graft osseointegration in the bone tunnel. Int Orthop 2011;35(10):1561-1567

25 Cho S, Li H, Chen C, Jiang J, Tao H, Chen S. Cationised gelatin and hyaluronic acid coating enhances polyethylene terephthalate artificial ligament graft osseointegration in porcine bone tunnels. Int Orthop 2013;37(03):507-513

26 Antonini S, Meucci S, Parchi P, et al. Human mesenchymal stromal cell-enhanced osteogenic differentiation by contact interaction with polyethylene terephthalate nanogratings. Biomed Mater 2016;11(04):045003 